\title{
Control externo de la calidad del diagnóstico serológico del dengue en laboratorios de países de las Américas, 1996-2001
}

\author{
María G. Guzmán, ${ }^{1}$ José L. Pelegrino, ${ }^{1}$ Tania Pumariega, ${ }^{1}$ Susana Vázquez, ${ }^{1}$ \\ Léster González, ${ }^{1}$ Gustavo Kouri ${ }^{1}$ y Jorge Arias $^{2}$
}

RESUMEN

Objetivo. Dar a conocer los resultados de cuatro controles externos de la calidad del diagnóstico serológico del dengue realizados en la Región de las Américas en el período de 1996- 2001 y presentar los resultados obtenidos por los laboratorios participantes.

Métodos. Se realizaron controles externos de la calidad del diagnóstico serológico del dengue en 1996-1997, 1998-1999, 1999-2000 y 2000-2001. Paneles compuestos por 20 sueros (12 de estos positivos a anticuerpos IgM contra el dengue) fueron enviados a laboratorios de la Región para que participaran en los controles realizados. Los sueros de los paneles eran negativos a anticuerpos contra el virus de la inmunodeficiencia humana y el virus de la hepatitis $C$, así como al antígeno de superficie de la hepatitis $B, y$ fueron almacenados a $-20{ }^{\circ} \mathrm{C}$ hasta su envío en refrigeración a los laboratorios participantes. La presencia de anticuerpos IgM se determinó mediante un ensayo inmunoenzimático (ELISA) de captura, mientras que el título de anticuerpos IgG se determinó por inhibición de la hemoaglutinación o ELISA de IgG. Los resultados de la determinación de anticuerpos IgM contra el dengue que no coincidian con los del centro de referencia se consideraron discordantes. El título de anticuerpos IgG se consideró discordante cuando los resultados difirieron en dos diluciones o más con respecto al resultado del centro de referencia.

Resultados. Un total de 27 laboratorios recibió 59 paneles de sueros de 1996 a 2001, y se recibieron los resultados del análisis de 54 de esos paneles (91,5\%). De 1080 sueros $(20 \times 54)$, $95,6 \%$ coincidieron con el laboratorio de referencia en cuanto a los resultados de la detección de anticuerpos IgM contra el dengue. Además, en 47 paneles (87\%) la coincidencia con el laboratorio de referencia se observó en $90 \%$ de las muestras del panel o más. De los 27 paneles para los cuales se recibieron los resultados de los títulos de anticuerpos IgG contra el dengue, $22(81,5 \%)$ coincidieron con el centro de referencia. Tomando en conjunto los cuatro controles realizados, 22 laboratorios coincidieron con el centro de referencia en $90 \%$ de las muestras o más y 13 en $100 \%$ de las muestras en cuanto a la presencia de IgM.

Conclusiones. Los resultados indican que la mayoría de los laboratorios participantes mostraron un excelente desempeño en la detección de anticuerpos IgG e IgM contra el dengue. No obstante, las deficiencias encontradas en algunos casos confirman la necesidad de seguir perfeccionando el diagnóstico de laboratorio del dengue en la Región.

Palabras clave Control analítico de calidad, diagnóstico serológico, dengue, ELISA, inmunoglobulina $\mathrm{M}$, inmunoglobulina $\mathrm{G}$.

Instituto de Medicina Tropical, Departamento de
Virología, Centro Colaborador OPS/OMS para
el Estudio de las Enfermedades Víricas, Ciudad
de La Habana, Cuba. La correspondencia debe ser
dirigida a: María G. Guzmán, Instituto de Medi-
cina Tropical "Pedro Kourí", Dpto. Virología,
Autopista Novia del Mediodía km 6, Ciudad Habana, Cuba. Teléfono: (53-7) 2020450, fax: (53-7) 204 6051. Correo electrónico: lupe@ipk.sld.cu.

2 Salud Ambiental, Condado de Fairfax, Virginia, EUA. Correo electrónico: jaria2@fairfaxcounty. gov.
Las últimas décadas han visto un aumento del número de epidemias de dengue y de dengue hemorrágico en el mundo. En la Región de las Américas, el número de países donde esta 
enfermedad se ha hecho endémica se ha incrementado, el vector -el mosquito Aedes aegypti- se ha extendido a nuevas áreas y los cuatro serotipos del virus han sido detectados en 19 países en los últimos 20 años (1). Esta situación se hace cada vez más grave y debido a la falta de una vacuna, las acciones de control del vector son las únicas que permiten incidir sobre la enfermedad (2).

En respuesta a esta situación, los países de la Región se han visto obligados a desarrollar sus capacidades de diagnóstico de laboratorio con miras a fortalecer la vigilancia clínica y epidemiológica. Actualmente, la mayoría de los países cuentan con al menos un laboratorio capaz de realizar el diagnóstico serológico del dengue y algunos pueden realizar el aislamiento viral o detectar su genoma mediante la reacción en cadena de la polimerasa (RCP). Finalmente, algunos países, como Argentina, Brasil, Colombia, México y Venezuela, han desarrollado redes de laboratorios para la vigilancia epidemiológica de esta enfermedad.

Durante la reunión del personal de los laboratorios que realizan el diagnóstico del dengue, efectuada en Río de Janeiro, Brasil en 1996, se subrayó la necesidad de mantener el control de la calidad del diagnóstico serológico y de extenderlo a nuevos laboratorios (3).

Como parte del apoyo que presta a los programas de prevención y control del dengue de los países de la Región, la Organización Panamericana de la Salud (OPS) organizó un programa de control externo de la calidad del diagnóstico serológico del dengue, con la participación de varios de sus centros colaboradores.

El objetivo de este trabajo es dar a conocer los resultados de cuatro controles externos de la calidad del diagnóstico serológico del dengue realizados en el período de 1996-2001 y presentar los resultados obtenidos por los laboratorios participantes.

\section{MATERIALES Y MÉTODOS}

El Departamento de Virología del Instituto de Medicina Tropical "Pedro Kourí" (IPK), Centro Colaborador de la
OPS/OMS para el Estudio de las Enfermedades Víricas, organizó y ejecutó en el período de 1996-2001 cuatro controles externos de la calidad del diagnóstico serológico del dengue. Se invitó a participar en estos controles (1996- 1997, 1998-1999, 1999-2000 y 2000- 2001) a las instituciones y laboratorios que tienen a su cargo la vigilancia serológica del dengue en los diferentes países de la Región, según una lista provista por la OPS. Posteriormente se incorporaron otros laboratorios interesados en participar en estos controles. En total se cursaron 86 invitaciones (de 18 a 24 invitaciones cada año).

Para la determinación de anticuerpos IgM contra el dengue, los laboratorios que participaron utilizaron el sistema inmunoenzimático de captura (MacELISA) (4), y en cuatro casos su variante ultramicroanalítica (UMELISA Dengue IgM, Centro de Inmunoensayo, Ciudad de La Habana, Cuba) (5).

Los paneles para el control de calidad se conformaron con sueros positivos de pacientes con dengue procedentes de Costa Rica, Nicaragua y Cuba, así como con sueros negativos de donantes de sangre de Cuba, previo consentimiento. A cada suero se le determinó la presencia de anticuerpos IgM contra el dengue mediante ELISA de captura (4) y el título de anticuerpos IgG mediante la prueba de inhibición de la hemoaglutinación (IH) (6) y ELISA de inhibición (7). Cada panel contenía 20 sueros, 12 de ellos positivos a anticuerpos IgM contra el dengue. Todos los sueros eran negativos a anticuerpos contra el virus de la inmunodeficiencia humana y el virus de la hepatitis $C$, así como al antígeno de superficie de la hepatitis B. Los sueros fueron almacenados a $-20{ }^{\circ} \mathrm{C}$ hasta su envío en refrigeración a los laboratorios participantes.

Los resultados obtenidos fueron informados a cada laboratorio de forma individual y confidencial.

Los resultados de la determinación de anticuerpos IgM contra el dengue que no coincidían con los del centro de referencia se consideraron discordantes. Los títulos de anticuerpos IgG se consideraron discordantes si diferían de los del centro de referencia en dos diluciones o más.

\section{RESULTADOS}

De las 86 invitaciones extendidas, 63 $(73,2 \%)$ fueron aceptadas. En cuatro ocasiones $(6,4 \%)$, los paneles de sueros no llegaron a su destino debido a dificultades de transporte. En conjunto se recibieron $54(91,5 \%)$ respuestas para su evaluación (cuadro 1).

Participó un total de 27 laboratorios de 17 países latinoamericanos (figura 1). De ellos, 3 participaron en los cuatro controles realizados, 5 lo hicieron en tres, 8 tomaron parte en dos, mientras que 11 laboratorios participaron en un solo control. En total, 16 laboratorios (59,3\%) participaron en dos o más de los cuatro controles realizados.

En relación con la determinación de anticuerpos IgM, en el período se estudiaron en total 1080 muestras y se observó una coincidencia general de $95,6 \%$ entre ellas y el centro de referencia. Al analizar la coincidencia

CUADRO 1. Participación de laboratorios en el control de calidad del diagnóstico serológico del dengue, por año, 1996-2001

\begin{tabular}{lccc}
\hline Período & $\begin{array}{c}\text { Paneles de sueros } \\
\text { enviados } \\
\text { (No.) }\end{array}$ & $\begin{array}{c}\text { Paneles de sueros } \\
\text { devueltos } \\
\text { (No.) }\end{array}$ & $\begin{array}{c}\text { Participación } \\
(\%)\end{array}$ \\
\hline $1996-1997$ & 18 & 12 & 66,7 \\
$1998-1999$ & 24 & 14 & 58,3 \\
$1999-2000$ & 20 & 17 & 85,0 \\
$2000-2001^{\text {a }}$ & 24 & 11 & 45,8 \\
Total & 86 & 54 & 62,8 \\
\hline a Cuatro laboratorios no recibieron los paneles de sueros.
\end{tabular}


FIGURA 1. Países y laboratorios que participaron en los controles de la calidad del diagnóstico serológico del dengue, 1996-2001

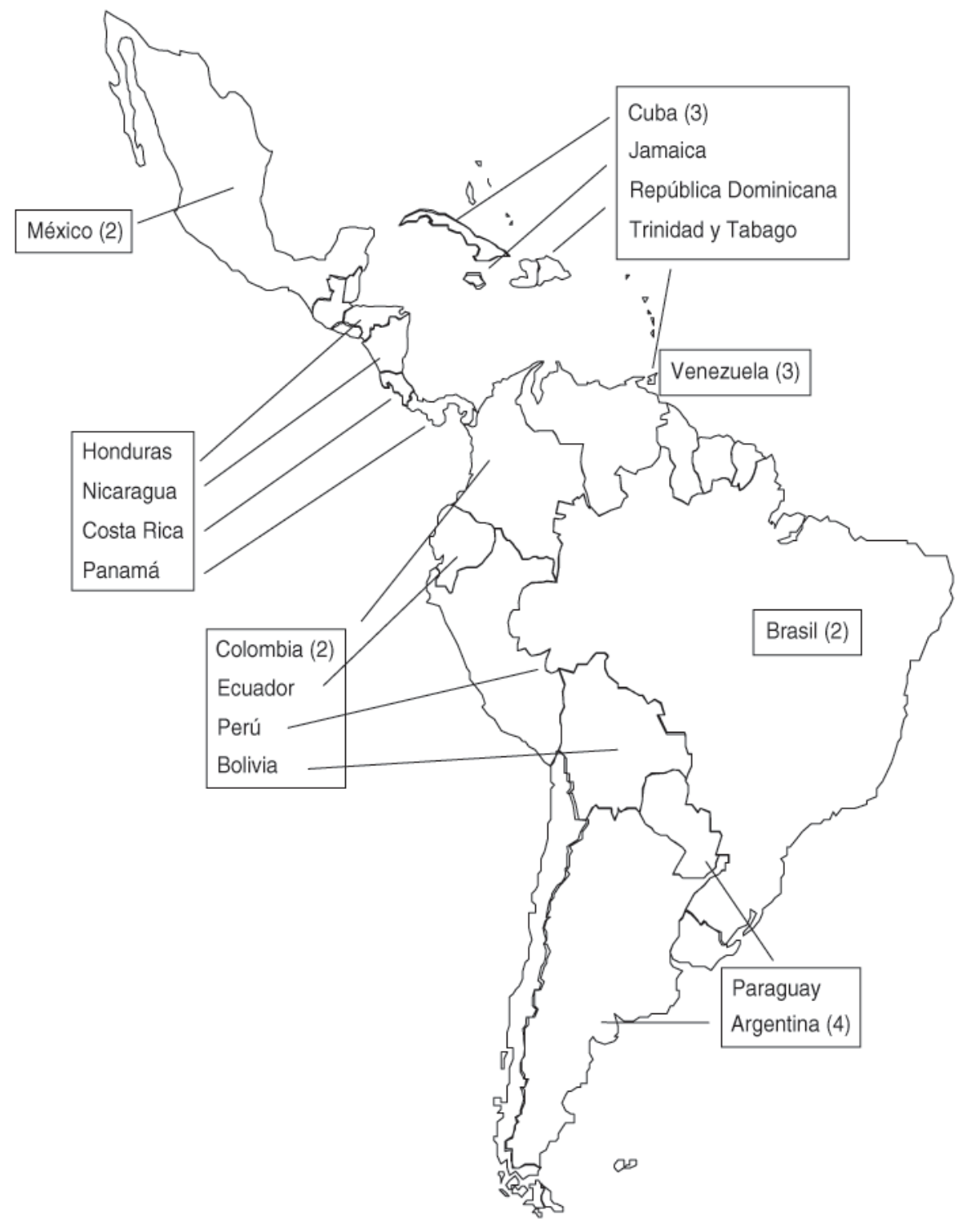

para cada laboratorio durante los cuatro controles realizados se observó que 13 laboratorios coincidieron en $100 \%$ de las muestras; 5 en $95-99 \%$; 4 en $90-94 \%$; 4 en $80-$ $89 \%$ y 1 en $65 \%$ (figura 2 ).

Los resultados de $70,4 \%$ de los laboratorios participantes coincidieron totalmente con los del centro de referencia en la detección de anticuerpos IgM. En 87\% (47/54) de los paneles, las respuestas coincidieron en $90 \%$ de las muestras del panel o más (cuadro 2). enfermedad. La peor situación se produjo en 2002, cuando se notificaron 1015420 casos, de los cuales 14374 se clasificaron como dengue hemorrágico. En total, 225 enfermos fallecieron.

Dada la gravedad de la situación, se hace impostergable mejorar los programas de control y prevención del dengue y fortalecer las bases epidemiológica y de laboratorio de la vigilancia de la enfermedad $(8,9)$. El diagnóstico de laboratorio debe permitir el seguimiento de los virus del dengue y conocer dónde hay transmisión viral, los serotipos y genotipos víricos involucrados y su asociación con los casos graves de la la enfermedad (9). Por ello, los países latinoamericanos han venido fortaleciendo su infraestructura analítica.

En 1996, 53 laboratorios en 14 países de la Región estaban equipados para realizar el diagnóstico serológico de la enfermedad, principalmente mediante la detección de anticuerpos IgM por ELISA de captura. De estos laboratorios, 20 contaban con 10 necesario para realizar el aislamiento viral y 11 poseían la capacidad para hacer el diagnóstico molecular (3).

Solo 26,8\% de los laboratorios invitados (1996-2001) declinaron participar en el estudio, debido a que participaban en controles externos organizados por otros centros colaboradores de la OPS/OMS.

Según los resultados obtenidos en los cuatro controles de la calidad realizados, $70,4 \%$ de los laboratorios coincidieron con el centro de referencia en los resultados de todos los sueros que formaban parte de los paneles para la determinación de anticuerpos IgM contra el dengue. Además, $87 \%$ de los
Solamente en 27 paneles se determinaron los títulos de IgG contra el dengue. De ellos, $22(81,5 \%)$ mostraron resultados coincidentes con los del centro de referencia (cuadro 3).

\section{DISCUSIÓN}

En las últimas décadas en la Región de las Américas ha habido un notable incremento del número de enfermos y de epidemias de dengue, así como de la extensión geográfica de la laboratorios lograron una coincidencia excelente o muy buena. Llama la atención que algunos laboratorios hayan conseguido una coincidencia elevada o máxima en los cuatro controles realizados, mientras que otros presentaron puntuaciones bajas repetidamente.

La mayor discordancia se observó en el control de la calidad de 19992000, período en el que se observaron tanto resultados negativos falsos como positivos falsos. Se debe señalar que 
FIGURA 2. Porcentaje de coincidencia en los resultados de anticuerpos IgM contra el dengue según el número de controles de la calidad en que participaron los laboratorios, 1996-2001

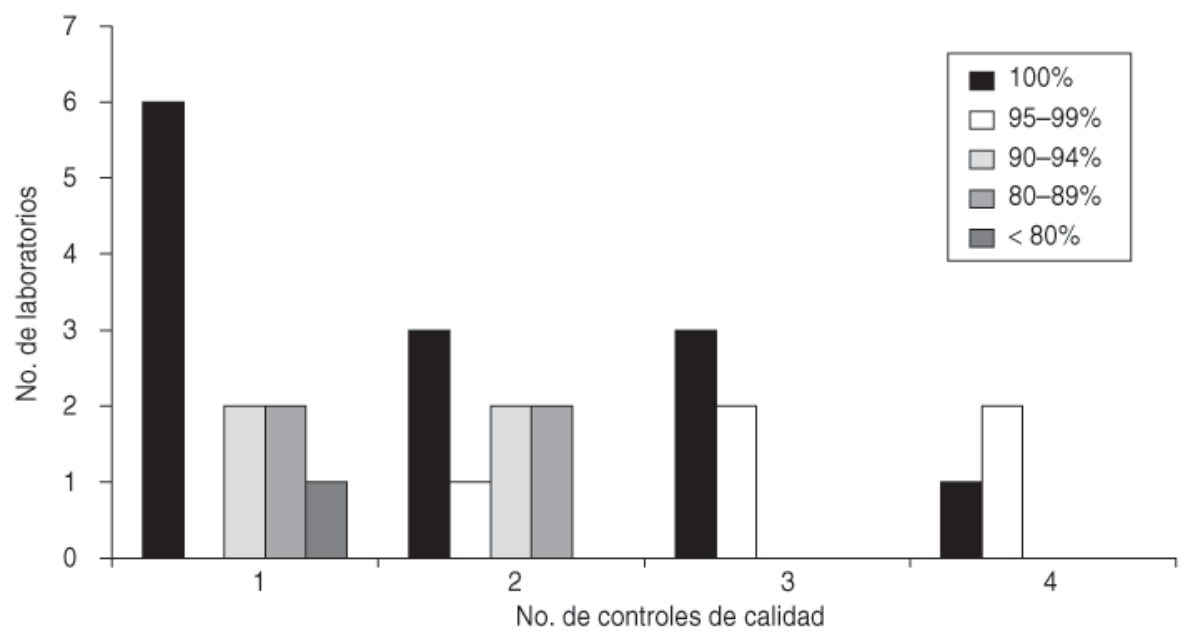

de los cuatro laboratorios con resultados discordantes en ese control, tres se incorporaban por primera vez a las pruebas y uno había presentado resultados discordantes en el control de 1996-1997. Los resultados discordantes en el control de la calidad de 2000-2001 se debieron a fallas en la detección de concentraciones bajas de anticuerpos IgM, en particular, en dos muestras cuyos valores de densidad óptica por ELISA no sobrepasaban en $10 \%$ el valor de corte de la prueba. Aunque no se puede descartar que esas muestras hayan sufrido una degradación parcial durante su almacenamiento o transporte, la explicación de esos resultados negativos falsos se debe atribuir a la baja sensibilidad de las técnicas de captura de IgM en algunos laboratorios, insuficiente para detectar concentraciones bajas de estos anticuerpos en el suero. Esto sugiere que algunos laboratorios deben mejorar aun más la calidad de su técnica de diagnóstico.

Los laboratorios encargados del diagnóstico serológico del dengue en la Región cuentan con varios años de experiencia realizando estas pruebas, por lo que se debe pasar a un nivel superior en la calidad de los controles. Es necesario evaluar cuidadosamente la sensibilidad de los sistemas utilizados, por lo que cada panel debe contener sueros con concentraciones bajas de anticuerpos IgM contra el dengue que permitan definir con mayor seguridad la sensibilidad real de los sistemas empleados y la capacidad de los laboratorios para detectar muestras de suero con bajos títulos de anticuerpos IgM. Esto es especialmente importante cuando se estudian muestras de pacientes que sufren una infección secundaria, en quienes las concentraciones de anticuerpos IgM son bajas o prácticamente indetectables. En este sentido, es muy importante garantizar las condiciones adecuadas de almacenamiento y de envío de los paneles de suero a los laboratorios participantes para evitar la degradación de las inmunoglobulinas.

Los controles de la calidad realizados han permitido detectar, además, algunas dificultades de los laboratorios nacionales y de referencia, entre ellas:

1. inestabilidad del suministro de antígenos, pues varios laboratorios dependen de los antígenos donados por los centros de referencia regionales

2. baja sensibilidad de las técnicas de captura de IgM

3. uso exclusivo de ELISA de captura de IgM para el diagnóstico serológico habitual, sin otra técnica serológica de apoyo al diagnóstico (a $50 \%$ de los paneles enviados no se les determinó la presencia de

CUADRO 2. Resultados de los paneles de sueros usados para el control de calidad de la determinación de anticuerpos IgM contra el dengue mediante ELISA o UMELISA de captura, 1996-2001

\begin{tabular}{|c|c|c|c|c|c|c|c|c|c|c|c|}
\hline \multirow[b]{3}{*}{ Período } & \multirow{3}{*}{$\begin{array}{c}\text { Paneles } \\
\text { de sueros } \\
\text { analizados }\end{array}$} & \multicolumn{10}{|c|}{ Coincidencia con el centro de referencia ${ }^{a}$} \\
\hline & & \multicolumn{2}{|c|}{ Excelente } & \multicolumn{2}{|c|}{$\begin{array}{c}\text { Muy } \\
\text { buena }\end{array}$} & \multicolumn{2}{|c|}{ Buena } & \multicolumn{2}{|c|}{ Regular } & \multicolumn{2}{|c|}{ Mala } \\
\hline & & No. & $(\%)$ & No. & $(\%)$ & No. & $(\%)$ & No. & $(\%)$ & No. & $(\%)$ \\
\hline 1996-1997 & 12 & 6 & $(50)$ & 3 & $(25)$ & 2 & $(16,7)$ & 1 & $(8,3)$ & 0 & \\
\hline 1998-1999 & 14 & 14 & $(100)$ & 0 & & 0 & & 0 & & 0 & \\
\hline 1999-2000 & 17 & 13 & $(76,5)$ & 0 & & 1 & $(5,9)$ & 2 & $(11,7)$ & 1 & $(5,9)$ \\
\hline 2000-2001 & 11 & 5 & $(45,5)$ & 6 & $(54,5)$ & 0 & & 0 & & 0 & \\
\hline Total & 54 & 38 & $(70,4)$ & 9 & $(16,6)$ & 3 & $(5,5)$ & 3 & $(5,5)$ & 1 & $(2,0)$ \\
\hline
\end{tabular}

a Los resultados representan el número de paneles de sueros que alcanzaron cada calificación. Entre paréntesis se indica el porcentaje con respecto al total de paneles que participaron en ese control. Excelente: coincidencia en 100\% de los sueros; muy buena: coincidencia en 90-99\%; buena: coincidencia en 80-89\%; regular: coincidencia en 70-79\%; mala: coincidencia en $69 \%$ o menos de los sueros. 
CUADRO 3. Resultados de la determinación de inmunoglobulinas totales o IgG contra el dengue mediante la prueba de inhibición de la hemoaglutinación o inmunoensayo enzimático (ELISA), según el año de evaluación, 1996-2001

\begin{tabular}{lcccc}
\hline & $\begin{array}{c}\text { Paneles de sueros } \\
\text { analizados }\end{array}$ & & \multicolumn{2}{c}{$\begin{array}{c}\text { Paneles con coincidencia en 100\% } \\
\text { de las determinaciones }\end{array}$} \\
\cline { 2 - 2 } Período & No. & & No. & $\%$ \\
\hline $1996-1997$ & 8 & & 7 & 87,5 \\
$1998-1999$ & 7 & 6 & 85,7 \\
$1999-2000$ & 10 & & 7 & 70,0 \\
$2000-2001$ & 2 & 2 & 100,0 \\
Total & 27 & 22 & 81,5 \\
\hline
\end{tabular}

anticuerpos totales o IgG contra el dengue).

Tomando en consideración la situación epidemiológica del dengue y del dengue hemorrágico en la Región de las Américas, se hace necesario seguir realizando controles externos de la calidad, incrementar su periodicidad e incorporar en ellos a un número mayor de laboratorios.

Los laboratorios podrían organizarse en una red que mantenga un estrecho y permanente intercambio con los centros colaboradores de la OPS/ OMS y con la OPS, lo que permitiría entrenar al personal periódicamente, utilizar protocolos estandarizados y únicos, controlar la calidad, suministrar reactivos de referencia, preparar bancos de sueros

1. Gubler DJ, Clark CG. Dengue/dengue hemorrhagic fever: the emergence of a global health problem. Emerg Infect Dis 1995;1:55-57.

2. Guzmán MG, Kourí G. Dengue: an update. Lancet Infect Dis 2002;2:33-42.

3. Pan American Health Organization, World Health Organization. Report of meeting of dengue laboratories in the Americas. Rio do Janeiro, Brazil, October 8, 1996. Washington, D.C.: PAHO; 1996. (PAHO/HCP/HCT/93/97).

4. Kuno G, Gomez I, Gubler DJ. An ELISA procedure for the diagnosis of dengue infection. J Virol Methods 1991;33:101-113.

5. Laferté J, Pelegrino JL, Guzmán MG, González G, Vázquez S, Hermida C. Rapid diagnosis of dengue virus infection using a novel $10 \mu \mathrm{L}$ IgM-antibody capture ultramicroELISA assay y cepas, efectuar investigaciones conjuntas e intercambiar información.

Sería muy conveniente extender el control de la calidad a los estudios virológicos (aislamiento viral e identificación mediante inmunofluorescencia) y moleculares (reacción en cadena de la polimerasa), mediante el intercambio de muestras de sueros obtenidos durante la fase aguda de la enfermedad. Estas técnicas se deben utilizar para confirmar el aislamiento viral e identificar el serotipo.

Tomando en cuenta el carácter emergente y reemergente del dengue en nuestra Región, así como de otras infecciones de importancia médica, se debe diseñar un sistema analítico que permita a los países enfrentar los nuevos retos epidemiológicos que pudieran avecinarse mediante el uso

\section{REFERENCIAS}

(MAC UMELISA Dengue). Adv Modern Biotechnol 1992;1:19.4.

6. Clark DH, Cassals J. Techniques for hemagglutination and hemagglutination inhibition with arthropod-borne virus. Am J Trop Med Hyg 1958;7:561-573.

7. Fernández RJ, Vázquez S. Serological diagnosis of dengue by an ELISA inhibition method (EIM). Mem Inst Oswaldo Cruz 1990;85:347351.

8. Pan American Health Organization. Dengue and dengue hemorrhagic fever in the Americas: guidelines for prevention and control. Washington, D.C.: PAHO; 1994. (Scientific Publication No. 548).

9. Organización Panamericana de la Salud, Organización Mundial de la Salud. La de la infraestructura existente y la incorporación de nuevas capacidades. Varios laboratorios tienen a su cargo la vigilancia de otras enfermedades de interés además del dengue - como el sarampión, la rubéola y la leptospirosis-, por lo que los controles de la calidad podrían ampliarse con muestras para evaluar también las técnicas utilizadas en el diagnóstico de esas infecciones.

\section{CONCLUSIONES}

Los resultados indican que la mayoría de los laboratorios que participaron en el estudio mostraron un excelente desempeño en la detección de anticuerpos IgG e IgM contra el dengue. No obstante, las deficiencias encontradas en algunos casos confirman la necesidad de seguir perfeccionando el diagnóstico de laboratorio del dengue en la Región.

Agradecimientos. Los autores desean agradecerles a todas las instituciones que participaron en este estudio su cooperación e interés en los resultados. También agradecen a aquellas que donaron sus muestras para la preparación de los paneles de sueros. Un agradecimiento especial al Dr. Francisco Pinheiro, de la Organización Panamericana de la Salud, quien fue el gestor de estos estudios. implementación del dengueNet en las Américas. Informe de la Reunión de OMS/OPS/CDC. San Juan, Puerto Rico, 9-11 de julio de 2002. Ginebra: OMS; 2003. (WHO/CDS/CSR/GAR 2003.8;PAHO/ $\mathrm{HCP} / \mathrm{HCT} / \mathrm{V} / 230 / 03)$.

Manuscrito recibido el 29 de enero de 2003. Aceptado para publicación, tras revisión, el 1 de octubre de 2003. 
ABSTRACT Objective. To report the results from participating laboratories for four external quality control proficiency tests of dengue serological diagnosis that were carried out in the Region of the Americas in the period of 1996-2001.

\section{Quality control of the serological diagnosis of dengue in laboratories throughout the Americas, 1996-2001}

Methods. External quality control proficiency tests of dengue serological diagnosis were carried out in 1996-1997, 1998-1999, 1999-2000, and 2000-2001. Panels made up of 20 serum samples (12 of them positive for dengue IgM antibodies) were sent to participating laboratories in the Region. The sera were negative for HIV antibodies, hepatitis $C$ virus antibodies, and hepatitis B surface antigen. The sera were stored at $-20^{\circ} \mathrm{C}$ until they were sent in refrigerated shipments to the participating laboratories. The presence of IgM antibodies was determined through IgM-capture enzyme-linked immunosorbent assay (ELISA), while the IgG antibody titer was determined by hemagglutination inhibition or by IgG ELISA. The results of the IgM antibody testing that differed from those of the reference center were considered discordant. The IgG antibody titer was considered discordant when the results differed by two dilutions or more with respect to the reference center's results.

Results. A total of 27 laboratories received a total of 59 serum panels over the 19962001 period, and the results from testing 54 of those panels $(91.5 \%)$ were sent back in. Of the total of 1080 sera samples from those 54 panels, the results from $95.6 \%$ of the $\operatorname{IgM}$ antibody tests were concordant with the results from the reference center. With 47 of the 54 panels $(87.0 \%)$ the participating laboratories' agreement with the reference center's results for the IgM antibody testing was $90.0 \%$ or higher. The laboratories sent back results from a total of $27 \mathrm{IgG}$ antibody titer tests, and 22 of them $(81.5 \%)$ coincided with those from the reference center. Considering the IgM antibody testing results from the four periods, the findings from 22 of the participating laboratories coincided with those from the reference center for at least $90 \%$ of the samples, and 13 of the laboratories were in complete concordance with the reference center.

Conclusions. The majority of the participating laboratories showed an excellent level of performance in detecting dengue $\operatorname{IgG}$ and $\operatorname{IgM}$ antibodies. However, the deficiencies found in some instances confirm the need for continuing to improve laboratory diagnosis of dengue in the Region of the Americas. 